\section{Literatur}

1. Shima H, Ikoma F, Terakawa T et al (1979) Developmental anomalies associated with hypospadias. J Urol 122:619-621

2. Ikoma F, Shima H, Yabumoto H (1985) Classification of enlarged prostatic utricle in patients with hypospadias. Br J Urol 57:334-337

3. Shima H, Ikoma F, Yabumoto H et al (1986) Gonadotropin and testosterone response in prepubertal boys with hypospadias. J Urol 135:539-542

4. Ikoma F, Shima H, Yabumoto H (1990) Caudal migration of verumontanum. Akt Urol 21(Suppl):74-79

5. Ikoma F, Shima H (1991) Caudal migration of verumontanum. J Pediatr Surg 26(7):858-861

6. Ikoma F (1994) Hypospadiekorrektor nach Denis Browne. In: Hohenfellner R (Hrsg) Ausgewaehlte Urologische OP-Techniken. Thieme, Stuttgart, S $4.77-4.90$

7. Schumacher S, Yabumoto H, Salge S et al (1997) Surgical result of complete hypopadias repair in two stages. Intn Urol Nephrol 29(3):333-340

8. Blandy JP, Lytton B (1986) What is the prostate and what is it for? In: Blandy JP, Lytton B (eds) The prostate. Butterworths, London, pp 1-11

9. Ikoma F, Shima H, Yabumoto H, Mori Y (1986) Surgical treatment for enlarged prostatic utricle and vagina musculina in patients with hypospadias. $\mathrm{Br}$ J Urol 58:423-428

10. Ikoma F (2007) Posterior sagittal approach in pediatric urology. In: Hohenfellner M, Santucci RA (eds) Emergencies in urology. Springer, Berlin Heidelberg New York, pp 541-547

Urologe 2014 · 53:1344-1349 • DOI 10.1007/s00120-014-3630-1

Online publiziert: 3. September 2014 • c C Springer-Verlag Berlin Heidelberg 2014

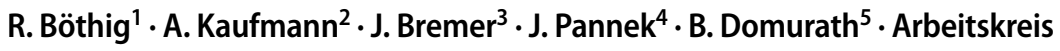
Neuro-Urologie der Deutschsprachigen Medizinischen Gesellschaft für Paraplegie (DMGP)

${ }^{1}$ Abteilung Neuro-Urologie, Querschnittgelähmtenzentrum,

Berufsgenossenschaftliches Unfallkrankenhaus Hamburg, Hamburg

${ }^{2}$ Zentrum für Kontinenz und Neuro-Urologie, Kliniken Maria Hilf Mönchengladbach, Mönchengladbach

${ }^{3}$ BDH-Klinik Greifswald, Greifswald

${ }^{4}$ Neuro-Urologie, Schweizer Paraplegiker-Zentrum, Nottwil

${ }^{5}$ Abteilung für Neuro-Urologie, Werner-Wicker-Klinik Bad Wildungen-Reinhardshausen, Bad Wildungen

\title{
Erratum zu: Botulinumneurotoxin Typ A bei neurogener Detrusorüberaktivität
}

\section{Konsensus des Arbeitskreises Neuro-Urologie der DMGP}

Entgegen der Angabe im Beitrag erfolgte die Erstbeschreibung zur Anwendung von Botulinumneurotoxin Typ A (BoNT-A) zur Dämpfung der neurogenen Detrusorhyperaktivität in der Publikation Stöhrer M, Schurch B, Kramer G, Schmid D, Gaul G, Hauri D (1999) Botulinum A-Toxin in the treatment of detrusor hyperreflexia in spinal cord injured patients: A new alternative to medical and surgical procedures? Neurourol Urodyn 18: 401-402.

Die Daten wurden auf dem ICSKongress 1999 in Denver präsentiert und im Rahmen der Kongressabstracts publiziert (doi: 10.1002/(SICI)1520-6777(1999)18:4<396::AIDNAU13>3.0.CO;2-K).

Wir bitten die korrekte Zitation zu beachten und den Fehler zu entschuldigen.

\section{Korrespondenzadresse}

\section{Dr. R. Böthig}

Abteilung Neuro-Urologie,

Querschnittgelähmtenzentrum, Berufsgenossenschaftliches Unfallkrankenhaus Hamburg

Bergedorfer Straße 10, 21033 Hamburg rboethig@gmx.de
Die Online-Version des Originalartikels können Sie unter: http://dx.doi.org/10.1007/s00120013-3419-7 finden. 